\title{
Terahertz Radiation Emission by Hot Electrons from AlGaN/GaN Heterostructure
}

\author{
V.A. Shalygin ${ }^{a}$, L.E. Vorobjev ${ }^{a}$, D.A. Firsov ${ }^{a}$, A.N. Sofronov ${ }^{a}$, G.A. Melentyev ${ }^{a} *$, \\ W.V. Lundin ${ }^{b}$, A.E. NikOlaev ${ }^{b}$, A.V. SAkharov ${ }^{b}$ And A.F. Tsatsulnikov ${ }^{b}$ \\ ${ }^{a}$ St. Petersburg State Polytechnic University, 195251 St. Petersburg, Russia \\ ${ }^{b}$ Ioffe Physical-Technical Institute, 194021 St. Petersburg, Russia
}

\begin{abstract}
In the present paper we report on the observation and study of intense spontaneous $\mathrm{THz}$ emission from modulation-doped AlGaN/GaN heterostructure under conditions of heating of two-dimensional electron gas in lateral electric field. The experimental results are compared with the model of blackbody-like thermal emission of hot 2D electrons. Complementary transport measurements and theoretical simulation were carried out to determine the dependence of effective electron temperature on electric field. The role of non-equilibrium optical phonon accumulation is discussed.
\end{abstract}

PACS: 73.50.Dn, 73.50.Fq, 78.60.Fi

\section{Introduction}

The development of effective and low-cost terahertz $(\mathrm{THz})$ emitters is widely acknowledged as essential for the advancement of terahertz diagnostic and information technology applications in medicine and biology, chemistry and physics, nanotechnology, and other areas. In the past decade, gallium nitride has attracted much attention for applications in the electrically pumped THz emitters (see [1-3] and references therein). In the present paper we report on the observation and study of spontaneous $\mathrm{THz}$ emission from modulation-doped $\mathrm{AlGaN} / \mathrm{GaN}$ heterostructures under conditions of two-dimensional (2D) electron gas heating in lateral electric field.

\section{Experimental details}

Heterostructure was grown by metal-organic vapor phase epitaxy (MOVPE) method on (0001) sapphire substrate using low-temperature GaN nucleation layer. The heterostructure represents a standard HEMT structure consisting of $4 \mu \mathrm{m}$ insulating GaN buffer, $1 \mathrm{~nm}$ AlN interface layer, $20 \mathrm{~nm}$ of modulation-doped $\mathrm{Al}_{0.3} \mathrm{Ga}_{0.7} \mathrm{~N}$ barrier, and $5 \mathrm{~nm}$ undoped GaN cap layer. For suppressing the formation of a conductive layer at $\mathrm{GaN} /$ sapphire interface, GaN nucleation layer was annealed under hydrogen-free ambience, which results in quasi-2D growth mode of high-temperature GaN buffer from the beginning of growth [4]. Growth regimes for top of GaN

* corresponding author; e-mail: gamelen@spbstu.ru buffer and AlN interface layer were optimized for suppression of interface erosion due to GaN-hydrogen interaction [4]. In accordance with measurements of conductivity and Hall effect performed by means of DC four-probe method, surface electron concentration at the AlGaN/ GaN interface is $n_{\mathrm{s}}=1.6 \times 10^{13} \mathrm{~cm}^{-2}$ with low-field mobility of 1500 and $5700 \mathrm{~cm}^{2} /(\mathrm{Vs})$ at the temperature of 300 and $4.2 \mathrm{~K}$, respectively.

Two Ti/Au electrical contacts of a few mm length with a distance of 1-7 $\mathrm{mm}$ were patterned on the top surface of every sample. To avoid overheating the samples, we used $2 \mu$ s voltage pulses with a repetition rate less than $1 \mathrm{~Hz}$ for transport measurements. The integral $\mathrm{THz}$ emission intensity was measured in the same pulse regime using a liquid-helium-cooled Ge:Ga photodetector equipped with a cold black polyethylene filter. The detector sensitivity range extended from 41 to $134 \mu \mathrm{m}$ (from 2.2 to $7.3 \mathrm{THz}$ ).

\section{Results and discussion}

Field dependence of $2 \mathrm{D}$ electron mobility in the lattice temperature range of 4.2-300 K (Fig. 1a) was determined from the experimental current-voltage characteristics assuming electron concentration invariability in the quantum well at the $\mathrm{AlGaN} / \mathrm{GaN}$ heterointerface. In low electric fields (less than $100 \mathrm{~V} / \mathrm{cm}$ ) the mobility is almost constant at every temperature. At lattice temperatures $T>100 \mathrm{~K}$, the value of low-field mobility rapidly decreases with temperature due to intensive electron scattering with optical phonon emission $[5,6]$. At fixed temperature, mobility decreases with electric field and the most significant decrease is observed at low temperatures when the low-field mobility is large enough to result in substantial 2D electron heating. 
Terahertz electroluminescence of 2D hot electron gas is more sensitive to the electric field effect than electron mobility. Integrated $\mathrm{THz}$ emission intensity increases about three orders of magnitude with the field growth from 25 to $2500 \mathrm{~V} / \mathrm{cm}$ (see Fig. 1b). In the same conditions the mobility change is less than one order of mag- nitude. As far as we know, here we report on the first experimental observation of $\mathrm{THz}$ radiation emission by hot electrons from AlGaN/GaN quantum wells. Similar investigations earlier were carried out on GaAs/AlGaAs quantum wells [7].
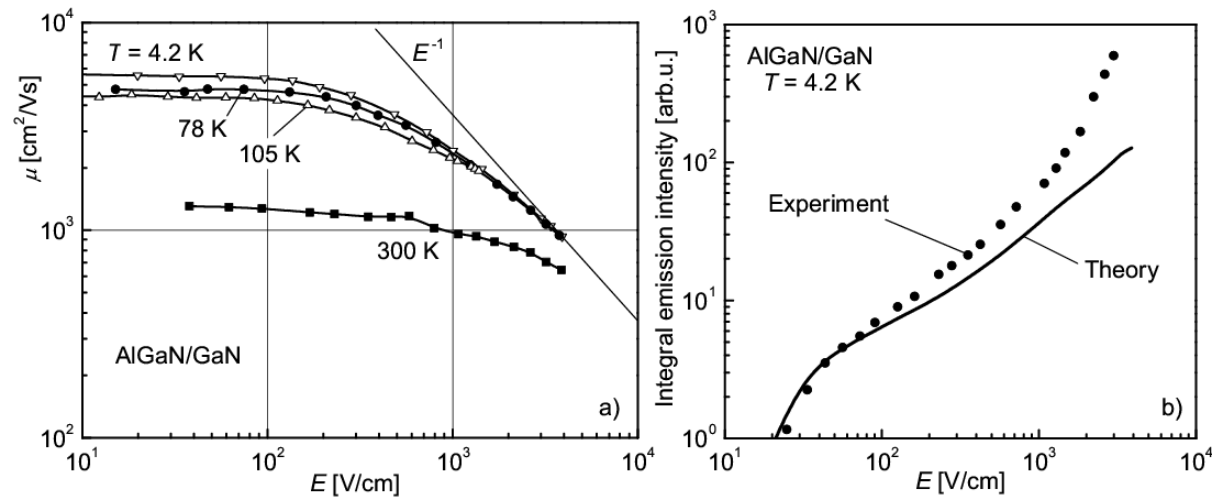

Fig. 1. (a) Field dependence of $2 \mathrm{D}$ electron mobility at different lattice temperatures $T$. (b) Field dependence of the integrated intensity of $\mathrm{THz}$ emission at $T=4.2 \mathrm{~K}$.
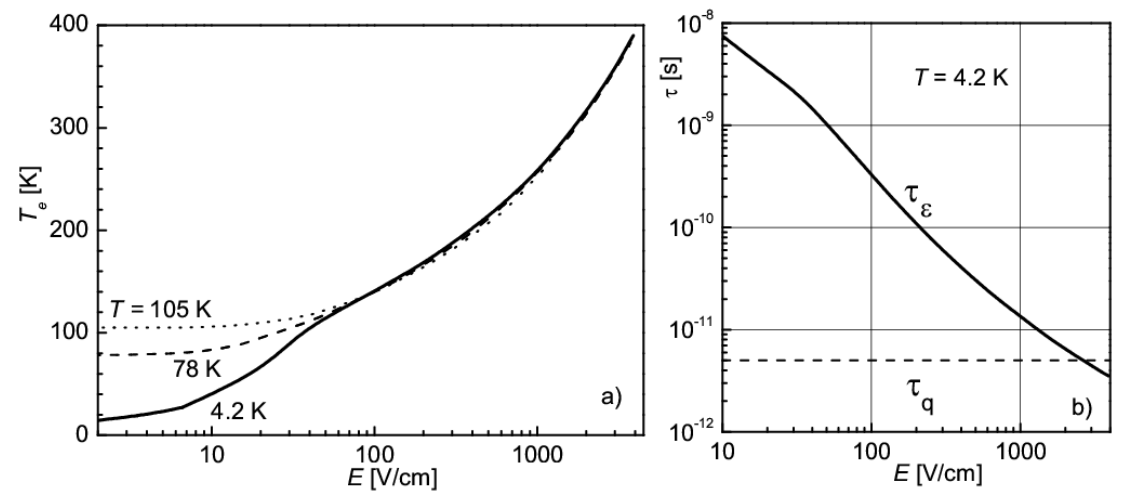

Fig. 2. (a) Field dependence of the hot electron temperature for different lattice temperatures. (b) Field dependence of the energy relaxation time $\tau_{\varepsilon}$ at $T=4.2 \mathrm{~K}$.

Let us consider $\mathrm{THz}$ radiation emission by hot electrons from $\mathrm{AlGaN} / \mathrm{GaN}$ quantum wells theoretically. A non-equilibrium distribution function $f(\varepsilon)$ of $2 \mathrm{D}$ electrons can be approximated by the shifted Fermi-Dirac distribution with a characteristic temperature $T_{\mathrm{e}}$. The field dependence of hot electron temperature $T_{\mathrm{e}}(E)$ can be found from the power balance equation

$$
e \mu E^{2}=\left\langle\frac{\mathrm{d} \varepsilon}{\mathrm{d} t}\right\rangle,
$$

where $\mu=\mu(E)$ is the field-dependent mobility of $2 \mathrm{D}$ electrons, and $\left\langle\frac{\mathrm{d} \varepsilon}{\mathrm{d} t}\right\rangle$ is the total energy loss rate per one carrier for all energy scattering mechanisms averaged over distribution function. To solve Eq. (1) we applied semiempirical method using dependence $\mu(E)$ from the experiment (Fig. 1a). The energy loss rate for the $i$-th scattering mechanism in 2D electron gas was described by integral [8]:

$$
\begin{aligned}
\frac{\mathrm{d} \varepsilon}{\mathrm{d} t} & =\int_{0}^{2 \pi} \mathrm{d} \theta \int_{-\infty}^{\infty} \mathrm{d} q_{z} \hbar \omega_{q}\left|I\left(q_{z}\right)\right|^{2} P_{i}\left(q_{x y}, q_{z}\right) f(\varepsilon) \\
\times & {\left[1-f\left(\varepsilon-\hbar \omega_{q}\right)\right] N_{q}(T) } \\
& \times\left[\exp \left(\frac{\hbar \omega_{q}}{k_{\mathrm{B}} T}\right)-\exp \left(\frac{\hbar \omega_{q}}{k_{\mathrm{B}} T_{\mathrm{e}}}\right)\right]
\end{aligned}
$$

where $q_{x y}$ and $q_{z}$ are phonon wave vector $\boldsymbol{q}$ components parallel and normal to interface, $\hbar \omega_{q}$ is the phonon energy, $\theta$ is the scattering angle, $N_{q}(T)$ is the phonon occupation number. The probability factors $P_{i}\left(q_{x y}, q_{z}\right)$ are determined in [9]. The form factor $\left|I\left(q_{z}\right)\right|^{2}$ was taken in the form $\left|I\left(q_{z}\right)\right|^{2}=\frac{b^{6}}{\left(b^{2}+q_{z}^{2}\right)^{3}}, b=\sqrt[3]{24 \pi m_{\mathrm{e}} n_{\mathrm{s}} e^{2} / \kappa_{0} \hbar^{2}}$ $[9,10]$, where $\kappa_{0}$ is the low-frequency dielectric constant of gallium nitride.. We calculated numerically $\left\langle\frac{\mathrm{d} \varepsilon}{\mathrm{d} t}\right\rangle$ for $2 \mathrm{D}$ electrons in $\mathrm{AlGaN} / \mathrm{GaN}$ quantum wells at $T=4.2$, 78 and $105 \mathrm{~K}$ taking into account (i) interaction with 
acoustic phonons via deformation potential coupling and piezoelectric coupling and (ii) interaction with optical phonons. The material parameters used for calculations were taken from Ref. [5].

In particular, for $T=4.2 \mathrm{~K}$ it was found that at $T_{\mathrm{e}}<100 \mathrm{~K}$ total energy loss rate is small enough because the dominant energy relaxation mechanism in this range is acoustic scattering. Processes of optical phonon emission begin to play a significant role at higher electron temperatures leading to rapid increase of total energy loss rate. Respectively one can see a rapid increase of $T_{\mathrm{e}}$ from $4.2 \mathrm{~K}$ to $100 \mathrm{~K}$ for electric field in the range 0-40 V/cm and limitation of $T_{\mathrm{e}}$ growth rate at higher fields (Fig. 2a). For the lattice temperature $T=105 \mathrm{~K}$ the change of the electron temperature $\Delta T_{\mathrm{e}}(E)=T_{\mathrm{e}}-T$ is smaller because at any electric field optical phonon emission plays dominant role.

The theoretical model of $\mathrm{THz}$ radiation emission by hot electrons from AlGaN/GaN quantum well can be developed after [7]. Following this paper, we apply the classical model of 2D electron gas conductivity $\sigma(\omega)=$ $\frac{e^{2} n_{\mathrm{s}} \tau_{\mathrm{p}}}{m_{\mathrm{e}}} \frac{1}{1-\mathrm{i} \omega \tau_{\mathrm{p}}}$ to calculate the emissivity of $2 \mathrm{D}$ electron system at AlGaN/GaN interface:

$$
A(\omega)=\frac{4 \operatorname{Re}\left[\sigma(\omega) / \varepsilon_{0} c\right]}{\left|1+\sqrt{\kappa_{\mathrm{s}}}+\sigma(\omega) / \varepsilon_{0} c\right|^{2}},
$$

where $\kappa_{\mathrm{S}}$ is the dielectric constant of the sapphire substrate and $\varepsilon_{0}$ is dielectric permittivity of vacuum. The experimental values of $\mu(E)$ were used to calculate the relaxation time $\tau_{\mathrm{p}}(E)=m_{\mathrm{e}} \mu(E) / e$. The spectral density of a blackbody-like radiation of $2 \mathrm{D}$ electron gas with a temperature $T_{\mathrm{e}}$ is given by the Planck distribution

$$
\begin{aligned}
& I\left(\omega, T_{\mathrm{e}}\right)=\int \frac{\hbar \omega^{3}}{4 \pi^{3} c^{2}\left[\exp \left(\hbar \omega / k_{\mathrm{B}} T_{\mathrm{e}}\right)-1\right]} \\
& \quad \times A(\omega) \cos (\vartheta) \mathrm{d} \Omega,
\end{aligned}
$$

where $\mathrm{d} \Omega$ is a solid angle element. Then the integral signal of the Ge:Ga photodetector response was simulated taking into account its sensitivity spectrum and the background radiation $I(\omega, T)$. Comparing the experimental dependence of integral intensity of the detected $\mathrm{THz}$ radiation on applied electric field with the calculated dependence (see Fig. 1b), one can conclude that the experimental results are qualitatively well described within the framework of the applied theory of blackbody-like thermal emission of hot $2 \mathrm{D}$ electrons.

However there is a significant quantitative discrepancy between considered theory and experiment in the range of high electric fields $(E>1000 \mathrm{~V} / \mathrm{cm})$. The discrepancy can be related to the non-equilibrium optical phonon accumulation which has not been taken into account in the simulation. Actually, the simulated energy relaxation time $\tau_{\varepsilon}$ decreases with the electric field (Fig. 2b) and at the certain value of electric field (about $1000 \mathrm{~V} / \mathrm{cm}$ ) becomes commensurable with the optical phonon lifetime $\tau_{q}=5 \times 10^{-12} \mathrm{~s}[11]$. Therefore at higher fields the optical phonon accumulation takes place.

\section{Conclusions}

Terahertz radiation emission by hot $2 \mathrm{D}$ electrons at the lattice temperature of $4.2 \mathrm{~K}$ was experimentally detected in the spectral range of $2.2-7.3 \mathrm{THz}$. The experimental data on the field dependence of the integral intensity of $\mathrm{THz}$ emission was compared with the theoretical simulation of blackbody-like thermal emission of hot $2 \mathrm{D}$ electrons. Experimental results are in a good qualitative agreement with considered theory. Significant quantitative discrepancy obtained at electric fields exceeding $1000 \mathrm{~V} / \mathrm{cm}$ can be attributed to the optical phonon accumulation.

\section{Acknowledgments}

This work was supported by Russian Federal Program "Kadry" for 2009-2013, RFBR, St. Petersburg Committee of Science and High Education, and the German-Russian Program of the Federal Ministry of Education and Research (BMBF).

\section{References}

[1] E. Starikov, P. Shiktorov, V. Gružinskis, L. Varani, C. Palermo, J.-F. Millithaler, L. Reggiani, J. Phys., Condens. Matter 20, 384209 (2008).

[2] A. El Fatimy, N. Dyakonova, Y. Meziani, T. Otsuji, W. Knap, S. Vandenbrouk, K. Madjour, D. Théron, C. Gaquiere, M.A. Poisson, S. Delage, P. Prystawko, C. Skierbiszewski, J. Appl. Phys. 107, 024504 (2010).

[3] V.A. Shalygin, L.E. Vorobjev, D.A. Firsov, V.Yu. Panevin, A.N. Sofronov, G.A. Melentyev, A.V. Antonov, V.I. Gavrilenko, A.V. Andrianov, A.O. Zakharyin, S. Suihkonen, P.T. Törmä, M. Ali H. Lipsanen, J. Appl. Phys. 106, 123523 (2009).

[4] W.V. Lundin, A.V. Sakharov, A.F. Tsatsulnikov, V.M. Ustinov, Semicond. Sci. Technol. 26, 014039 (2011).

[5] M. Shur, B. Gelmont, M. Asif Khan, J. Electron. Mater. 25, 777 (1996).

[6] Z. Dziuba, J. Antoszewski, J.M. Dell, L. Faraone, P. Kozodoy, S. Keller, B. Keller, S.P. DenBaars, U.K. Mishra, J. Appl. Phys. 82, 2996 (1997).

[7] K. Hirakawa, M. Grayson, D.C. Tsui, C. Kurdak, Phys. Rev. B 47, 16651 (1993).

[8] Cz. Jasiukiewicz, V. Karpus, Semicond. Sci. Technol. 11, 1777 (1996).

[9] K. Hirakawa, H. Sakaki, Phys. Rev. B 33, 8291 (1986).

[10] A.M. Kurakin, S.A. Vitusevich, S.V. Danylyuk, H. Hardtdegen, N. Klein, Z. Bougrioua, A.V. Naumov, A.E. Belyaev, J. Appl. Phys. 105, 073703 (2009).

[11] K.T. Tsen, R.P. Joshi, D.K. Ferry, A. Botchkarev, B. Sverdlov, A. Salvador, H. Morkoc, Appl. Phys. Lett. 68, 2990 (1996). 